EPJ Web of Conferences 108, 02040 (2016)

DOI: $10.1051 /$ epjconf/201610802040

C Owned by the authors, published by EDP Sciences, 2016

\title{
A New Model for the Mechanochemical Corrosion of a Thin Spherical Shell
}

\author{
O. Sedova ${ }^{1, a}$ and Y. Pronina ${ }^{1, b}$ \\ ${ }^{1}$ Saint Petersburg State University, 7-9, Universitetskaya nab., St.Petersburg, 199034, Russia
}

\begin{abstract}
A pressurized thin-walled spherical shell under the conditions of double-sided uniform corrosion is considered. The rates of corrosion are supposed to dependent linearly on the effective stress. Previous solutions for thin shells obtained by other authors reflect only the effect of differential pressure (i.e., the difference between internal and external pressure). The model proposed here allows to include also the effect of hydrostatic pressure on the durability of the shell under the corrosion conditions.
\end{abstract}

\section{Introduction}

Corrosion is a process of material destruction that occurs as a result of chemical interaction between a material and its environment. The most common type of corrosion damages the entire surface of the material at about the same rate, and, as a consequence, results in a failure. If a structural element, immersed in an aggressive environment, is subject to mechanical stresses, then the so-called mechanochemical effect may occur (i.e. corrosion accelerated by stress) [1,2].

Many scholars have studied the stress state of structural members under mechanochemical corrosion conditions. In the case when the corrosion is understood as a uniform dissolution, there are a few works reporting closed-formed expressions, see, e.g., [3-9]. Stress-assist chemical reactions sustained by diffusion in deformable solids were modeled in [10-13]. Initial boundary value problems with evolving boundaries have to be solved for other types of wear [14-16] and in other areas [17]. Because of the complexity of such problems, most of the research was done using numerical methods.

In this paper an analytical solution is presented for a thin-walled spherical shell subject to internal and external pressure under the conditions of double-sided uniform corrosion. The rates of corrosion are supposed to dependent linearly on the effective stresses. Previous solutions for thin shells [4] do not depend on the internal and external pressures themselves, but only on their difference (see [18]). However, the hydrostatic pressure can affect the corrosion rate [1]. Accurate solutions for the doublesided corrosion of shells under internal and external pressure based on the solutions of Lamé problems for thick cylinder and sphere are rather cumbersome [19-25]. The model proposed here is simpler but, it allows to take into account the effect of the hydrostatic pressure on the durability of the thin shells under the mechanochemical corrosion.

\footnotetext{
a e-mail: ag.olya.sedova@gmail.com

be-mail: y.pronina@spbu.ru
} 


\section{Problem formulation}

Consider a linearly elastic thin-walled sphere with the inner $r$ and outer $R$ radii subjected to dead internal $p_{r}$ and external $p_{R}$ pressure (figure 1). The sphere is uniformly corroding from the inside and the outside with the dissolution rates $v_{r}$ and $v_{R}$, respectively. The corrosion process affects the shell sizes: the inner radius increases while the outer radius decreases from the initial time $t_{0}$ values denoted respectively by $r_{0}$ and $R_{0}$.

According to $[1,3]$, the rates of corrosion on the inner and outer surfaces are:

$$
v_{r}=\frac{d r}{d t}=a_{r}+m_{r} \sigma\left(r, R, p_{r}, p_{R}\right), \quad v_{R}=-\frac{d R}{d t}=a_{R}+m_{R} \sigma\left(r, R, p_{r}, p_{R}\right),
$$

where the constants $a_{r}, a_{R}, m_{r}$, and $m_{R}$ are determined experimentally [1]. In general, they are different under tension and compression; $\sigma\left(r, R, p_{r}, p_{R}\right)$ is the maximum principal stress assumed to be identical through the entire thickness.

We suppose that at any time $t$ the maximum principal stress $\sigma$ (which is a circumferential stress) depends on the instantaneous values of the inner $r(t)$ and outer $R(t)$ radii of the shell and on the given constant pressures $p_{r}$ and $p_{R}$. The problem is to find the time dependence of the maximum stress at any $t>t_{0}$.

Consider a spherical coordinate system $(\rho, \theta, \phi)$ with the origin identical to the center of the hollow sphere. In previous solutions obtained by other authors (for example, [4]), $\sigma$ was chosen as

$$
\sigma\left(r, R, p_{r}, p_{R}\right)=\sigma_{\phi \phi}=\sigma_{\theta \theta}=\frac{\left(p_{r}-p_{R}\right) r_{c}}{2 h},
$$

where $r_{c}=(R+r) / 2$ is the radius of the middle surface of the spherical shell, $h=R-r$ is the thickness of the shell. Naturally, the solution derived using (2) does not depend on the pressure values $p_{r}$ and $p_{R}$, but only on the difference $p_{r}-p_{R}$. However, it is known that the hydrostatic pressure $p=\min \left\{p_{r}, p_{R}\right\}$ could affect the corrosion kinetics [1]. To take into account the effect of the hydrostatic pressure $p$ we suppose that the effective stress is defined by the more accurate formula [26]:

$$
\sigma=\sigma\left(r, R, p_{r}, p_{R}\right)=\sigma_{\phi \phi}=\sigma_{\theta \theta}=\frac{\left(p_{r}-p_{R}\right) r_{c}}{2 h}-q,
$$

where $q=\left(p_{r}+p_{R}\right) / 2$.

In both equations (2) and (3), the radius $r_{c}$ is assumed to remain constant, while the thickness $h$ is changing with the time.

\section{Problem solution}

Adding Eqs. (1) 1 and (1) 2 (with negative signs), we obtain

$$
\frac{d(R-r)}{d t}=\frac{d h}{d t}=-(a+m \sigma)
$$

where $a=a_{r}+a_{R}, m=m_{r}+m_{R}$.

Differentiating (3) with respect to $t$ and using (4) yields the ordinary differential equation:

$$
\frac{d \sigma}{d t}=\frac{2(a+m \sigma)(\sigma+q)^{2}}{\left(p_{r}-p_{R}\right) r_{c}}
$$




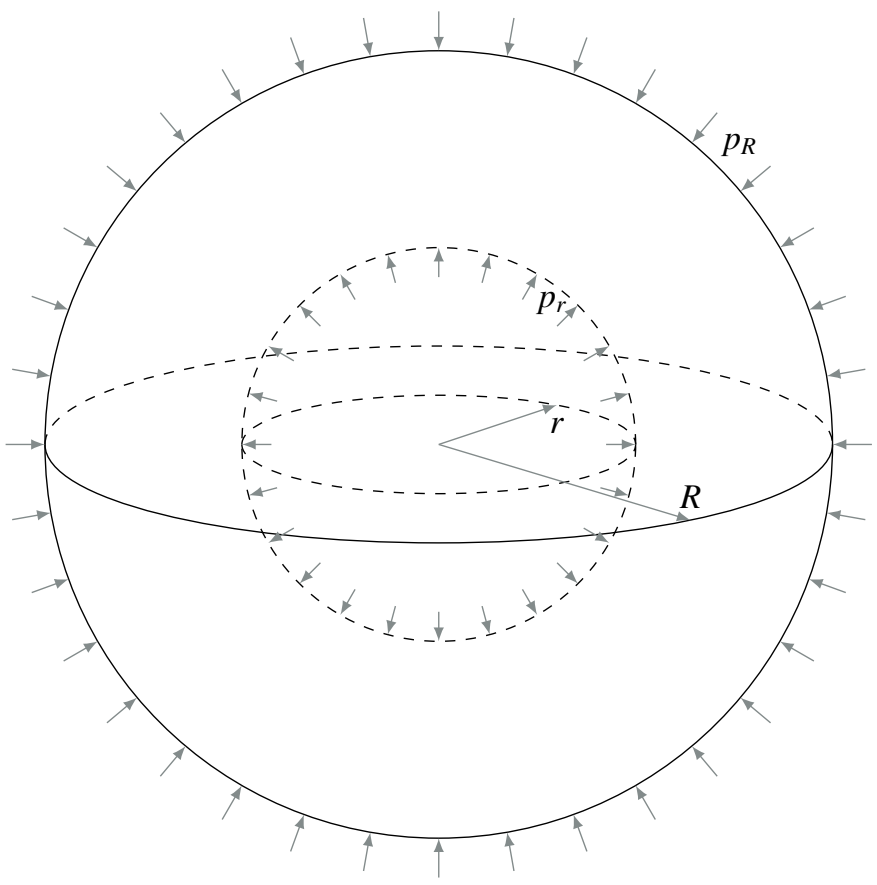

Figure 1. The model of a pressurised hollow sphere

The initial condition to be satisfied at $t=t_{0}$ is

$$
\left.\sigma\right|_{t=t_{0}}=\sigma_{0}=\frac{\left(p_{r}-p_{R}\right) r_{c}}{2 h_{0}}-q
$$

where $h_{0}=R_{0}-r_{0}$. The solution of (5), with the above initial condition is

$$
t=t_{0}+\frac{\left(p_{r}-p_{R}\right) r_{c}}{2(a-m q)}\left(\frac{m}{a-q m} \ln \frac{(a+m \sigma)\left(\sigma_{0}+q\right)}{\left(a+m \sigma_{0}\right)(\sigma+q)}+\frac{\sigma-\sigma_{0}}{(\sigma+q)\left(\sigma_{0}+q\right)}\right) .
$$

It is known that a higher hydrostatic pressure may lead to an increase in the corrosion pitting growth probability. Note that for spherically and cylindrically symmetric problems, analytical solutions can often be obtained [27-29, etc.]. In the case of a more complex geometry of the solid (especially, made of nonlinear materials), it is necessary to use numerical methods [30-32]. Some results concerning the stress distribution near corrosion pits in spherical shells are presented in $[33,34]$.

\section{Comparison with other solutions}

Let us compare calculation results obtained by the proposed model (using Eq. (3) as the effective stress) with the results based on both the classical thin shell theory formula (Eq. (2) is used as the effective stress) and the Lamé's formula for a thick pressurised spherical shell [23, 24]. In figure 2 the dependencies of $|\sigma|$ on $t$ are demonstrated for different corrosion models of the spherical shell with the initial radii $R_{0}=100\left[l_{c}\right]$ and $r_{0}=96\left[l_{c}\right] ; t_{0}=0$. Here, $l_{c}$ is a certain length unit. Other corrosion 


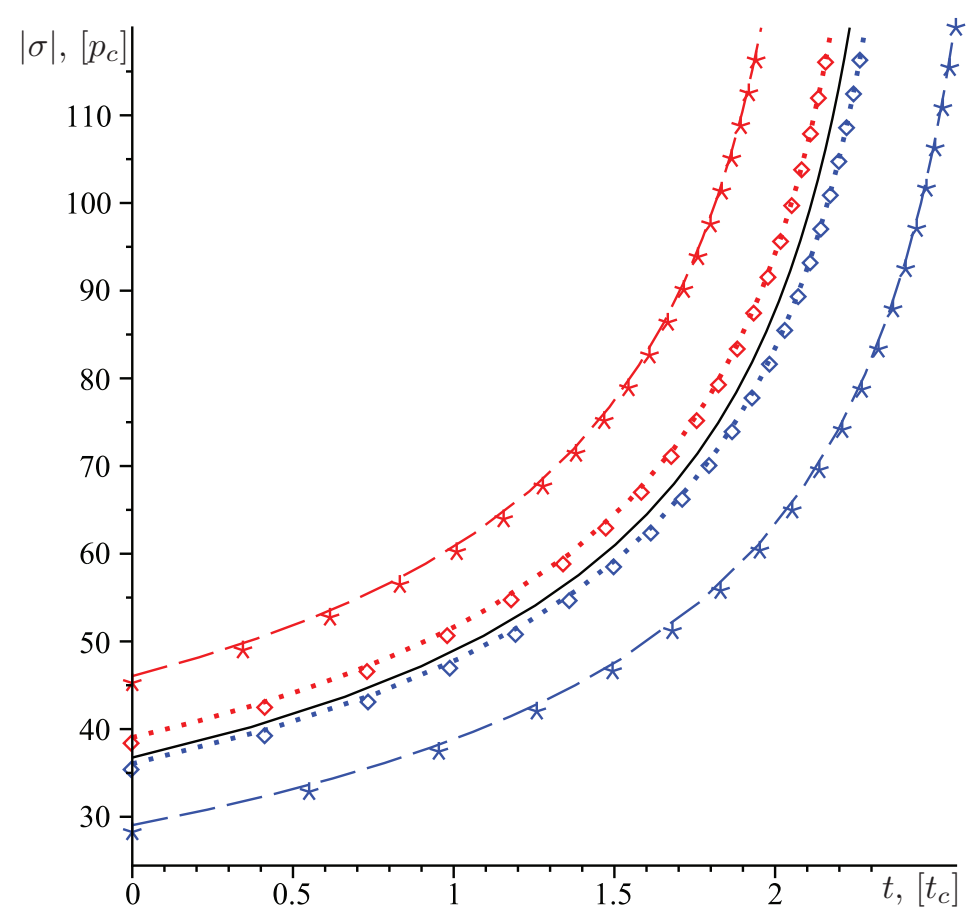

Figure 2. Dependencies of $|\sigma|$ on the time $t$ for various pressure values

rate parameters are $a_{r}=a_{R}=0.16\left[l_{c} / t_{c}\right]$ and $\left.m_{r}=m_{R}=0.008\left[l_{c} /\left(t_{c} p_{c}\right)\right]\right)$. Here and below, $t_{c}$ is an appropriate unit of time; $p_{c}$ is a given unit of stress.

In order to observe the effect of hydrostatic pressure $p=\min \left\{p_{r}, p_{R}\right\}$, four sets of the internal and external pressure values have been used:

- $p_{r}=3\left[p_{c}\right], p_{R}=0$ (blue dotted line and diamonds);

- $p_{r}=9\left[p_{c}\right], p_{R}=6\left[p_{c}\right]$ (blue dashed line and asterisks);

- $p_{r}=0, p_{R}=3\left[p_{c}\right]$ (red dotted line and diamonds);

- $p_{r}=6\left[p_{c}\right], p_{R}=9\left[p_{c}\right]$ (red dashed line and asterisks).

These sets are chosen such that, despite various values of the pressures, the absolute value of the pressure difference remains constant: $\left|p_{r}-p_{R}\right|=3\left[p_{c}\right]$.

It is obvious that, under an effective stress given by (2), the dependencies of $|\sigma|$ on the time $t$ for all the above sets of pressure values are the same. In other words, for the given parameters, the classical thin shell theory formula provides only one curve; it is the black curve in figure 2.

For the other two models we have different curves for each of the considered sets of pressure values. Red curves in figure 2 correspond to the case when $p_{r}-p_{R}<0$, while blue ones correspond to positive differences, $p_{r}-p_{R}>0$. Dotted and dashed curves indicate the dependencies $|\sigma(t)|$ obtained in $[23,24]$ by the means of the Lamé's formula for hydrostatic pressures $p=0$ and $p=6$, respectively. Diamonds and asterisks are used for the model proposed here at the corresponding pressure values. 
The figure shows that the curves for all three models are close to each other at zero hydrostatic pressure, $p=\min \left\{p_{r}, p_{R}\right\}=0$ (compare solid line, dotted lines and diamonds). However, the difference between the classical thin shell model (solid line) and two other models (dashed lines and stars) increases with the growing $p$. At the same time, the curves corresponding to the accurate formulas [23] and to the proposed thin shell model are hardly different from each other at any pressure values. A good agreement between these two models is also observed even for a thick shell (with thickness relation $\left.h / r_{c}=1 / 5\right)$. In the framework of our accurate models it was shown that the durability of the sphere increases as $p$ grows if $p_{r}-p_{R}>0$, and decreases if $p_{r}-p_{R}<0$.

\section{Conclusion}

A new mathematical model for the mechanochemical corrosion of thin spherical shells was defined. A linearly elastic sphere subject to internal $p_{r}$ and external $p_{R}$ pressure, and uniformly corroding from the inside and outside has been considered. The rates of corrosion are supposed to be linearly dependent on the effective stress. An analytical solution of the problem has been obtained.

The computational results for the classical thin shell model and for the proposed here refined model are very close to each other for zero hydrostatic pressure $p=\min \left\{p_{r}, p_{R}\right\}=0$, but the difference between them grows as $p$ increases. It has been shown that the durability of the sphere increases as $p$ grows if $p_{r}-p_{R}>0$, and decreases if $p_{r}-p_{R}<0$. The solutions based on the Lamé's formula and that proposed here are hardly different from each other at any pressure values.

\section{Acknowledgements}

The work has been done with partial support of International Innovative Nanotechnology Centre of the CIS countries (InINCIS) grant 080-316. Calculations have been performed using the computing resources of the resource center "SPbU Computing Facility".

\section{References}

[1] P. A. Pavlov, B. A. Kadyrbekov and V. V. Borisevich, Soviet Materials Science 21 (3), 248 (1985)

[2] E. M. Gutman, Mechanochemistry of solid surfaces (World Scientific, Singapore, 1994) 322

[3] V. M. Dolinskii, Chemical and Petroleum Engineering 3 (2), 96 (1967)

[4] V. G. Karpunin, S. I. Kleshchev and M. S. Kornishin, Proceedings, 10th All-Union Conference of the Theory of Shells and Plates 1, 166 (1975). (In Russian)

[5] I. G. Ovchinnikov and Yu. M. Pochtman, Soviet Materials Science 27 (2), 105 (1992)

[6] R. M. Bergman, S. P. Levitsky, J. Haddad and E. M. Gutman, Thin-Walled Struct. 44 (7), 726 (2006)

[7] A. I. Rusanov, Russ. J. Gen. Chem. 77 (4), 491 (2007)

[8] Y. G. Pronina, Russian Metallurgy (Metally) 2010 (4), 361 (2010)

DOI: $10.1134 /$ S0036029510040208

[9] I. Elishakoff, G. Ghyselinck and Y. Miglis, Journal of Applied Mechanics, Trans. ASME 79 (2), 021013 (2012)

[10] A. B. Freidin, ASME International Mechanical Engineering Congress and Exposition, Proceedings (IMECE) 9 (2013)

[11] A. B. Freidin, E. N. Vilchevskaya and I. K. Korolev, International Journal of Engineering Science 8357 (2014). DOI: 10.1016/j.ijengsci.2014.03.008 
[12] E. N. Vilchevskaya, A. B. Freidin and N. F. Morozov, Doklady Physics 60 (4), 175 (2015)

[13] A. M. Lokoshchenko and A. V. Sokolov, Mechanics of Solids 49 (1), 49 (2014). DOI: 10.3103/S0025654414010063

[14] I. Paczelt, S. Kucharski and Z. Mroz, Wear 274-275, 127 (2012)

[15] I. Paczelt and Z. Mroz, Key Engineering Materials 618, 49 (2014)

[16] I. Paczelt and Z. Mroz, Advances in Engineering Software 80 (C), 139 (2015)

[17] I. V. Zhukova, E. P. Kolpak and Yu. E. Balykina, Applied Mathematical Sciences 8 (29-32), 1455 (2014). DOI: 10.12988/ams.2014.4135

[18] Y. G. Pronina, O. S. Sedova and S. A. Kabrits, AIP Conference Proceedings 1648, 300008 (2015). DOI: $10.1063 / 1.4912550$

[19] Y. G. Pronina, Proceedings of the 4th International Conference on Computational Methods for Coupled Problems in Science and Engineering, COUPLED PROBLEMS 2011, 1408 (2011)

[20] Y. Pronina, International Journal of Solids and Structures 50 (22-23), 3626 (2013). DOI: 10.1016/j.ijsolstr.2013.07.006

[21] Y. G. Pronina, Computational Plasticity XII: Fundamentals and Applications - Proceedings of the 12th International Conference on Computational Plasticity - Fundamentals and Applications, COMPLAS 2013, 729 (2013)

[22] Y. G. Pronina, Corrosion Science 90, 161 (2015). DOI: 10.1016/j.corsci.2014.10.007

[23] O. Sedova and Y. Pronina, Mechanics Research Communications 65, 30 (2015). DOI: 10.1016/j.mechrescom.2015.02.007

[24] O. S. Sedova and Y. G. Pronina, AIP Conference Proceedings 1648, 260002 (2015). DOI: 10.1063/1.4912519

[25] O. S. Sedova and Y. G. Pronina, 2015 International Conference on Mechanics - Seventh Polyakhov's Reading (2015) 7106771. DOI: 10.1109/POLYAKHOV.2015.7106771

[26] Y. M. Dahl, Vestnik Sankt-Peterburgskogo Universiteta. Ser. 10. Prikladnaya Matematika. Informatika. Protsessy Upravleniya 4, 55 (2009)

[27] N. A. Gasratova, APRN Journal of Engineering and Applied Sciences, 9 (11), 2259 (2014)

[28] Y. G. Pronina, Mechanics of Solids 49 (3), 302 (2014). DOI: 10.3103/S0025654414030066

[29] S. Bauer, E. Voronkova, Shell and Membrane Theories in Mechanics and Biology, Advanced Structured Materials 45, H. Altenbach and G. I. Mikhasev (editors), 79 (2015). DOI: 10.1007/978-3-319-02535-3_14

[30] S. A. Kabrits, E. P. Kolpak and K. F. Chernykh, Mechanics of solids 21, 182 (1986)

[31] N. L. Bochkareva and E. P. Kolpak, Vestnik Sankt-Peterburgskogo Universiteta. Ser 1. Matematika Mekhanika Astronomiya 4, 49 (1993)

[32] S. A. Kabrits and E P. Kolpak, AIP Conf. Proc. 1648, 300005 (2015). DOI: 10.1063/1.4912547

[33] O. S. Sedova, L. A. Khaknazarova and Y. G. Pronina, 2014 10th International Vacuum Electron Sources Conference, IVESC 2014 and 2nd International Conference on Emission Electronics, ICEE 2014 - Proceedings, 1 (2014)

[34] O. S. Sedova, Emission Electronics (ICEE), 2014 2nd International Conference on IEEE, 1 (2014). DOI: 10.1109/Emission.2014.6893977 